Check for updates

Cite this: Soft Matter, 2019, 15,6362

Received 3rd June 2019

Accepted 9th July 2019

DOI: $10.1039 / c 9 s m 01112 a$

rsc.li/soft-matter-journal

\title{
Transient measurement and structure analysis of protein-polysaccharide multilayers at fluid interfaces
}

\author{
Pascal Bertsch, (iD *a Alexandra Thoma, ${ }^{a}$ Jotam Bergfreund, (ID) ${ }^{a}$ Thomas Geue (iD ${ }^{b}$ \\ and Peter Fischer iD *a
}

\begin{abstract}
The formation of electrostatic protein-polysaccharide multilayers has attracted attention for the design of fluid interfaces with enhanced stability and functionality. However, current techniques are often limited to measuring final multilayer properties. We present an interfacial shear rheology setup with simultaneous subphase exchange, allowing the transient measurement of biopolymer multilayers by their viscoelasticity. The successive and simultaneous adsorption of $\beta$-lactoglobulin $(\beta-\mathrm{lg})$ and low-methoxyl pectin were investigated at the $n$-dodecane/water interface at $\mathrm{pH} 4$. The successive injection of pectin increased the viscoelasticity of an adsorbed $\beta$-lg layer by electrostatic complexation. On the other hand, simultaneous adsorption impeded adsorption kinetics and interfacial layer strength due to complexation in the bulk phase prior to adsorption. Neutron reflectometry at the air-water interface confirmed the formation of an initial $\beta$-lg layer and electrostatic complexation of a secondary pectin layer, which desorbed upon $\mathrm{pH}$-induced charge inversion. The layer formed by simultaneous adsorption mainly consisted of $\beta$-lg. We conclude that protein-polysaccharide complexes show limited surface activity and result in a lower effective protein concentration available for adsorption.
\end{abstract}

\section{Introduction}

The stabilization of fluid interfaces by proteins is a common approach in colloid and interface science. Proteins adsorb at the air/water $(\mathrm{A} / \mathrm{W})$ or oil/water $(\mathrm{O} / \mathrm{W})$ interface, induce a decrease in interface tension, and form a viscoelastic interfacial network. ${ }^{1}$ The successive adsorption of a polysaccharide has emerged as a promising approach to improve the performance of protein stabilized interfaces, such as increased resistance against heat and ionic strength, ${ }^{2,3}$ applicability in a broad $\mathrm{pH}$-range,,$^{3,4}$ increased interfacial moduli, ${ }^{5}$ or stability under gastric conditions. $^{6,7}$

Here, we use $\beta$-lactoglobulin ( $\beta$-lg), a globular protein fractionated from whey. ${ }^{8}$ The adsorption of globular proteins at fluid interfaces comprises three consecutive subprocesses: (i) diffusion limited transport to the interface, ${ }^{9}$ (ii) conformational changes and adsorption limited by the proteins' thermodynamic stability, ${ }^{1,10}$ and (iii) interfacial rearrangement. ${ }^{11}$ The functional structure of $\beta$-lg regarding interactions with lipids is

\footnotetext{
${ }^{a}$ Institute of Food Nutrition and Health, ETH Zurich, 8092 Zurich, Switzerland. E-mail: pascal.bertsch@hest.ethz.ch,peter.fischer@hest.ethz.ch; Tel: +41446328536

${ }^{b}$ Laboratory of Neutron Scattering and Imaging, Paul Scherrer Institut, 5232 Villigen PSI, Switzerland
}

a hydrophobic $\beta$-strand barrel called calyx. ${ }^{12}$ Because of the affinity of the calyx towards hydrophobic substances, the adsorption of $\beta$-lg strongly depends on the hydrophobicity of the employed subphase, as shown in molecular dynamics simulations ${ }^{13}$ and experimentally. ${ }^{14} \beta$-lg has a $\mathrm{pH}$-dependent charge with an isoelectric point $\approx 5$, where it forms the most compact interfacial layers due to minimum intermolecular repulsion. ${ }^{15}$

Most polysaccharides are not surface active and do not adsorb at fluid interfaces. An established approach is the adsorption of a primary protein layer followed by electrostatic complexation of a secondary polysaccharide layer, as employed here. Alternatively, proteins and polysaccharides may be covalently conjugated. ${ }^{16-18}$ In case of electrostatic complexation, the formation of a stable protein layer prior to polysaccharide addition is crucial, as complexation in the bulk impedes adsorption kinetics and results in weaker interfacial layers compared to pure protein layers.,19 On the other hand, the successive adsorption of a polysaccharide at an equilibrated protein layer increases interfacial layer strength, as demonstrated by Ganzevles et al. ${ }^{5}$ by an increase in interfacial shear moduli. However, interfacial rheology measurements are usually restricted to measuring final layer properties, stressing the need for transient interfacial rheology measurements during multilayer formation.

Here, we employ an interfacial shear rheology setup with simultaneous subphase exchange. This allowed the formation 
of pure $\beta$-lg layers followed by injection of low-methoxyl pectin while measuring interfacial shear rheology. The interfacial viscoelasticity during simultaneous and successive injection of $\beta$-lg and low-methoxyl pectin was investigated and complemented with dynamic interface tension, interfacial dilatation, and neutron reflectivity measurements.

\section{Materials and methods}

\subsection{Materials}

$\beta$-lg was obtained from the Food and Bioprocess Engineering group at the Technical University of Munich, Germany. ${ }^{8}$ Lowmethoxyl pectin (32\% degree of esterification (DE)) was kindly provided by CP Kelco, Germany. Citric acid monohydrate was purchased from Fisher Scientific, Switzerland. Disodium hydrogen phosphate, $n$-dodecane, and $\mathrm{NaOH}$ were purchased from Sigma Aldrich, Switzerland. HCl (37\% fuming) was purchased from Merck, Switzerland. Milli-Q water was obtained from a Merck Millipore system, Germany.

Experiments were performed at $\mathrm{pH} 4$ in 1/10 McIlvaine buffer mixed from $10 \mathrm{mM}$ citric acid and $20 \mathrm{mM}$ disodium hydrogen phosphate. The protein or pectin was added to the buffer an the $\mathrm{pH}$ adjusted using $1 \mathrm{M} \mathrm{HCl}$ and $\mathrm{NaOH}$, respectively. The zeta-potential of $\beta$-lg and pectin as a function of $\mathrm{pH}$ was determined by a Zetasizer nano ZS (Malvern, U.K.).

\subsection{Methods}

2.2.1 Interfacial shear rheology with simultaneous subphase exchange. An Anton Paar MCR 501 (Graz, Austria) was equipped with a bicone geometry ${ }^{20}$ and a subphase exchange cup. $^{21}$ The bicone was placed at the air-buffer interface and covered with $n$-dodecane. A time-sweep at $1 \mathrm{rad} \mathrm{s}^{-1}$ angular frequency and $0.5 \%$ strain was initiated at the clean $\mathrm{O} / \mathrm{W}$ interface followed by the injection of $50 \mathrm{~mL} 0.1 \mathrm{wt} \% \beta-\mathrm{lg}$ solution at $0.5 \mathrm{~mL} \mathrm{~min}^{-1}$ after $5 \mathrm{~min}$. The same volume was subtracted to keep the interface level. The delayed injection prevents protein adsorption at the $\mathrm{A} / \mathrm{W}$ interface prior to oil addition and allows the transient measurement of protein adsorption kinetics by an increase in dynamic moduli $G_{\mathrm{i}}{ }^{\prime}$ and $G_{\mathrm{i}}{ }^{\prime \prime} \cdot{ }^{14}$ After $24 \mathrm{~h}, 20 \mathrm{~mL} 0.1 \mathrm{wt} \%$ pectin suspension were injected at $0.5 \mathrm{~mL} \mathrm{~min}^{-1}$. An Ismatec Vario 7337-00 peristaltic pump (Cole Parmer, Vernon Hills, USA) with a flow rate of $6 \mathrm{~mL} \mathrm{~min}^{-1}$ was connected to the exchange cup to provide constant mixing. The protein solution was injected from $60 \mathrm{~mL} \mathrm{BD}$ luer-lok syringes (Becton Dickinson, USA) with a syringe pump PHD 2000 (Harvard Apparatus, USA). The pectin suspension was injected from $20 \mathrm{~mL}$ B. Braun syringes (Switzerland) with an AL1000-220 syringe pump (World Precision Instruments, Germany). The use of smaller syringes was found to be crucial for the secondary injection to prevent protein layer breakage, probably due to a smoother startup. For the combined injection, $50 \mathrm{~mL}$ containing each $0.1 \mathrm{wt} \% \quad \beta-\mathrm{lg}$ and pectin were injected from the same $60 \mathrm{~mL}$ BD luer-lok syringe at $0.5 \mathrm{~mL} \mathrm{~min}^{-1}$. Experiments were performed in 1/10 McIlvaine buffer at $\mathrm{pH} 4$ and $20{ }^{\circ} \mathrm{C}$.
2.2.2 Dynamic interface tension measurement. A drop profile tensiometer PAT-1 (SINTERFACE Technologies, Germany) was employed to assess the dynamic interfacial tension of an $n$-dodecane drop formed at a U-shaped capillary. The drop contour was monitored by a CCD camera and the interfacial tension determined by axisymmetric drop shape analysis. The oil drop was formed in $0.001 \mathrm{wt} \% \beta$-lg solution followed by injection of $2 \mathrm{~mL}$ pectin suspension after $16 \mathrm{~h}$, or in a mixture of each $0.001 \mathrm{wt} \% \beta$-lg and pectin. Experiments were performed in 1/10 McIlvaine buffer at $\mathrm{pH} 4$ and $22{ }^{\circ} \mathrm{C}$.

2.2.3 Interfacial dilatational rheology. A Wilhelmy-plate setup (KSV Nima, Finland) was equipped with a Teflon trough $(15 \times 7.8 \times 0.5 \mathrm{~cm})$ built in house with a $2 \mathrm{~cm}$ Teflon barrier on each side, resulting in a surface area of $86 \mathrm{~cm}^{2}$. The through was filled with $65 \mathrm{~mL} \mathrm{1/10} \mathrm{McIlvaine} \mathrm{buffer} \mathrm{at} \mathrm{pH} 4$ and the barriers were fully closed to check for contaminations. The surface pressure was determined by a paper plate $21 \mathrm{~mm}$ in perimeter. Either pure $\beta$-lg solution, a $\beta$-lg-pectin mixture, or pure $\beta$-lg solution followed by a pectin suspension after $4 \mathrm{~h}$ were injected. After surface pressure equilibration, interfacial dilatational experiments were performed by sinusoidal oscillation of the Teflon barriers at $10 \mathrm{mHz}$ parallel to the plate. The interfacial dilatational storage modulus $E^{\prime}$ was calculated from 25 normalized oscillation cycles using MATLAB (R2008a). The room temperature was controlled to $22{ }^{\circ} \mathrm{C}$.

2.2.4 Neutron reflectivity. Neutron reflectivity experiments were performed at the Swiss Spallation Neutron Source SINQ, Paul Scherrer Institute, Switzerland, with the AMOR time-of-flight reflectometer. $^{22,23}$ The same Langmuir-trough and Wilhelmyplates as elaborated above were used and filled with $65 \mathrm{~mL}$ 1/10 McIlvaine buffer prepared in $\mathrm{D}_{2} \mathrm{O}$ at $\mathrm{pD} 4$. The neutron reflection at pure buffer was recorded as a reference. Neutron reflection of a protein film formed at $0.001 \mathrm{wt} \% \beta$-lg was determined, followed by injection of $0.002 \mathrm{wt} \%$ pectin to assess the structure of successively adsorbed multilayers. For comparison, a layer formed during simultaneous adsorption of each 0.005 wt $\%$-lg and pectin was analyzed. Reflection experiments were started $6 \mathrm{~h}$ after biopolymer injection. The neutron reflection was recorded at three angles of incidence $\theta$ $(0.5,1.3$, and 2.8$)$ at varying wavelength $\lambda(3.5$ to $12 \AA)$, thereby covering a $Q$-range of $0.01-0.187 \AA^{-1}$. The experiment at the first angle of incidence $(\theta=0.5)$ was repeated at the end to verify that the layers did not undergo structural changes. The temperature was controlled to $20{ }^{\circ} \mathrm{C}$. Obtained reflectivity data was fitted using the Parratt algorithm. ${ }^{24}$ As scattering length density $1.78 \times 10^{-6}$ and $2.47 \times 10^{-6} \AA^{-2}$ were used for $\beta$-lg and pectin, respectively. Fitted reflectivity curves had a $\chi^{2}$ below $7.5 \times 10^{-3}$.

\section{Results and discussion}

\subsection{Electrostatic complexation of $\beta$-lg and pectin}

As proteins and most polysaccharides have a pH-dependent charge, the $\mathrm{pH}$ is crucial in order to form electrostatic proteinpolysaccharide complexes. Fig. 1 depicts the zeta-potential, the magnitude of electrostatic potential, for $\beta$-lg and pectin as 


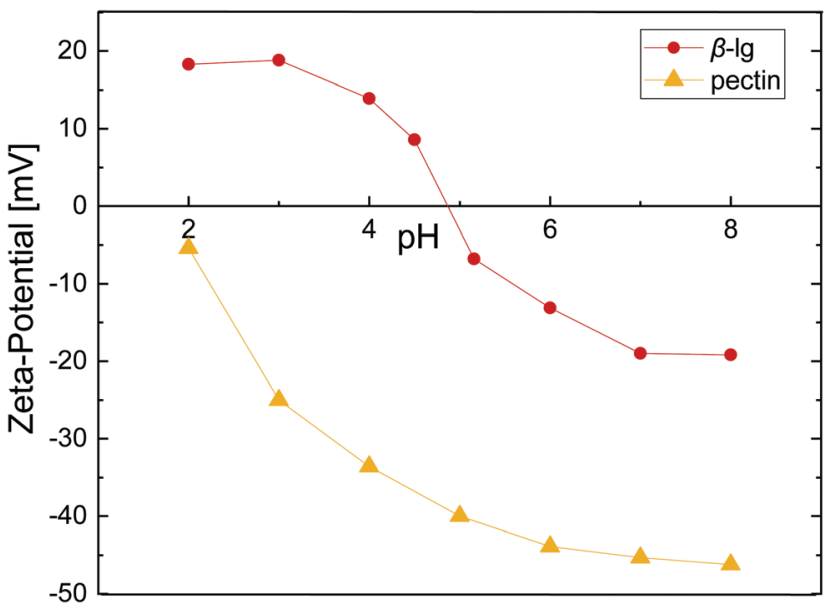

Fig. 1 Zeta-potential as a function of $\mathrm{pH}$ for $1 \mathrm{wt} \% \beta$-lg and $0.1 \mathrm{wt} \%$ pectin in 1/10 Mcllvaine buffer. Lines are to guide the eye.

a function of $\mathrm{pH} . \beta$-lg is charged negatively at neutral $\mathrm{pH}$ and shows a charge inversion at the isoelectric point $\approx 4.9$. The charge of pectin on the other hand derives from its carboxyl groups, and can thus only be charged negatively. The $\mathrm{p} K_{\mathrm{a}}$ of the carboxyl groups is $\approx 3,{ }^{25}$ resulting in a decreasing net charge at low $\mathrm{pH}$. To form electrostatic interfacial multilayers, all further experiments were performed at $\mathrm{pH} 4$ where the zeta-potential of $\beta$-lg and pectin is +14 and -34 , respectively. A low-methoxyl pectin $(32 \% \mathrm{DE})$ was chosen to have a higher number of potentially charged carboxyl groups and increase the proteinpectin binding affinity. The electrostatic complexation of $\beta$-lg and pectin as a function of $\mathrm{pH}$ and pectin $\mathrm{DE}$ was addressed in detail by Girard et $a l^{26}$ An association constant of $1.4 \times$ $10^{3} \mathrm{M}^{-1}$ was reported at $\mathrm{pH} 4$ for a comparable pectin with $28 \% \mathrm{DE}$. The formation of electrostatic $\beta$-lg-pectin complexes is reversible. ${ }^{27}$ Such electrostatic complexes may be exploited to manipulate the charge in protein stabilized emulsions, yielding emulsion droplets with negative surface charge in a broad pH-range. ${ }^{2,4}$

\subsection{Transient interfacial rheology measurement during multilayer formation}

The adsorption of proteins is a common approach to stabilize fluid interfaces. As indicated above, the successive adsorption of a polysaccharide can increase the stability and functionality of adsorbed protein layers. Dynamic interface tension measurements are commonly employed to assess the formation of interfacial layers, as shown in Fig. 2A. $\beta$-lg adsorbs at the $n$-dodecane/water interface and decreases its interface tension to $20 \mathrm{mN} \mathrm{m}^{-1}$. The successive adsorption of pectin had a minor impact on surface tension as it is not surface active itself. On the other hand, in case of simultaneous adsorption $\beta$-lgpectin complexes are formed in the bulk prior to adsorption, resulting in slower adsorption kinetics and a higher final surface tension compared to pure $\beta$-lg. Although these measurements are useful for the determination of adsorption kinetics and changes in interface tension, they fail to detect the adsorption of non-surface active polysaccharides, and neglect the potential increase in viscoelasticity upon polysaccharide adsorption.

We employed an interfacial shear rheology setup with simultaneous subphase exchange, ${ }^{21}$ allowing the successive injection of $\beta$-lg and pectin while measuring interfacial shear rheology. This method was recently demonstrated to allow transient measurements of dynamic interfacial moduli $G_{\mathrm{i}}{ }^{\prime}$ and $G_{\mathrm{i}}{ }^{\prime \prime}$ during $\beta$-lg adsorption. ${ }^{14}$ Fig. 2B depicts the interfacial layer formation of pure $\beta$-lg with successive pectin addition compared to their simultaneous injection. The injection of $50 \mathrm{~mL} 0.1 \mathrm{wt} \% \beta-\mathrm{lg}$ solution $\left(0.5 \mathrm{~mL} \mathrm{~min}^{-1}\right)$ was initiated after $5 \mathrm{~min}$ and a measurable viscoelastic network was formed after $20 \mathrm{~min}$. Note that a peristaltic pump was attached to provide constant mixing, resulting in a significantly shorter lag-phase compared to our previous investigation of diffusion limited $\beta$-lg adsorption. ${ }^{14} \mathrm{After}$ 24 hours, $20 \mathrm{~mL} 0.1 \mathrm{wt} \%$ pectin were injected to the equilibrated $\beta$-lg layer, resulting in a twofold increase in interfacial moduli. Thus, the electrostatic complexation of pectin at an adsorbed $\beta-\mathrm{lg}$ layer increases its interfacial viscoelasticity. This is in contrast to simultaneous injection of $\beta$-lg and pectin. The electrostatic complexation in the bulk prior to adsorption results in a prolonged lag-phase, slower adsorption, and lower $G_{\mathrm{i}}{ }^{\prime}$ and $G_{\mathrm{i}}{ }^{\prime \prime}$ compared to the pure $\beta$-lg layer. The adverse effect of proteinpolysaccharide complexation on adsorption kinetics is in agreement with dynamic interface tension measurements and was reported before. ${ }^{5,19}$ It is attributed to the slower diffusion, lower affinity for adsorption, or even precipitation of the $\beta$-lg-pectin complexes. In our case with constant mixing diffusion probably played a minor role, underlining the importance of the latter two. The adsorption limiting effect of polysaccharides is enhanced at increasing polysaccharide charge density, ${ }^{28}$ and may be counteracted by increasing the $\beta$-lg/pectin ratio. ${ }^{5}$

Fig. 2C depicts strain sweep experiments of the formed biopolymer layers. The mixed $\beta$-lg-pectin layer formed after simultaneous adsorption showed the lowest critical strain with yield at $1 \%$. Pure $\beta$-lg and the successively adsorbed $\beta$-lg-pectin layer showed similar critical strains at $\approx 2.7 \%$. Thus, the additional adsorption of pectin increases the dynamic interfacial moduli without lowering the critical strain, which is often observed for layers at increasing interfacial moduli. ${ }^{7}$ An overview of equilibrated $G_{\mathrm{i}}{ }^{\prime}$ and critical strain of the different biopolymer layers is provided in Fig. 2D. Compared to pure $\beta$-lg, $G_{\mathrm{i}}{ }^{\prime}$ was increased twofold by the successive adsorption of pectin. Comparable results were obtained for the successive adsorption of other proteins and polysaccharides under associative conditions. ${ }^{16,29,30}$ The interfacial elasticity of successively adsorbed $\beta-\lg$ and pectin was five times higher compared to simultaneous adsorption, in good agreement with findings of Ganzevles et $a .^{5}$ who found up to sixfold stronger interfacial layers. We used a $\beta$-lg/pectin mixing ratio of $1: 1$. Ganzevles et al. ${ }^{5}$ found that at $\beta-\mathrm{lg} /$ pectin ratios higher than five the adverse effects of pectin on adsorption kinetics and interfacial viscoelasticity diminish. For successive adsorption on the other hand, the mixing ratio does not affect layer properties. In contrast to our results, the complexation of longer protein fibrils and pectin in 

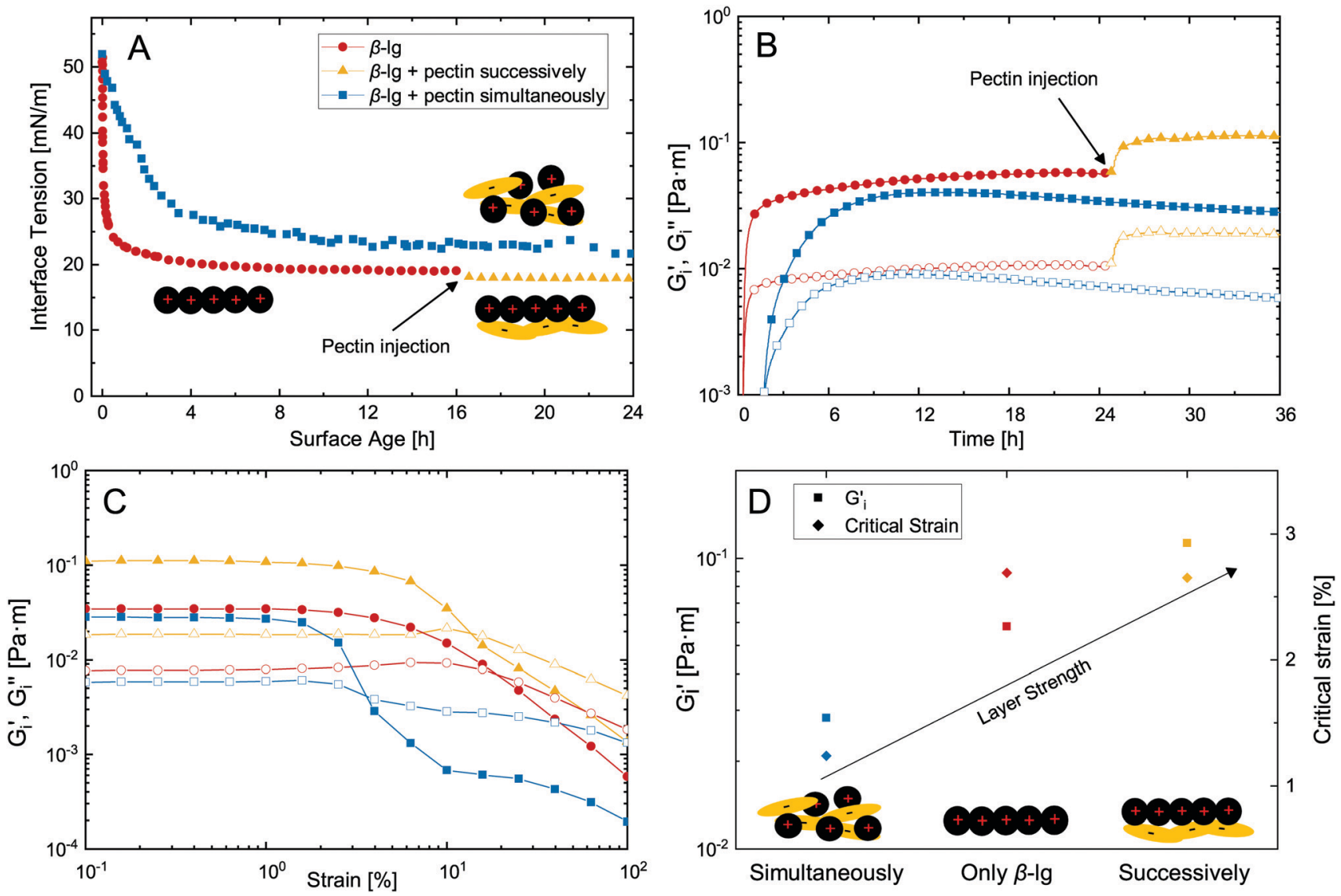

Fig. 2 (A) Dynamic interface tension measurements of an $n$-dodecane droplet formed in $1 / 10$ Mcllvaine buffer at pH 4 with 0.001 wt $\% \beta$-lg and 0.01 wt\% pectin added before drop formation or after $16 \mathrm{~h}$. (B) Interfacial shear rheology measurements at the $n$-dodecane/water interface with simultaneous subphase exchange showing interfacial storage $G_{i}^{\prime}$ (full) and loss modulus $G_{i}^{\prime \prime}$ (empty) as a function of time during injection of $\beta$-lg with successive pectin injection or a $\beta$-lg/pectin mixture. (C) Strain sweep experiments and (D) equilibrium $G_{i}^{\prime}$ and critical strain of the formed biopolymer layers. Experiments were performed at $1 \mathrm{rad} \mathrm{s}^{-1}$ angular frequency, $0.5 \%$ strain, and $20{ }^{\circ} \mathrm{C}$ in $1 / 10 \mathrm{Mcllvaine}$ buffer at $\mathrm{pH} 4$. Lines are to guide the eye.

the bulk may even increase the interfacial viscoelasticity compared to pure protein fibril layers. ${ }^{31}$ The presence of polysaccharides may also increase the strength of protein layers at neutral $\mathrm{pH}$ when both are charged negatively. This is attributed to a limited thermodynamic compatibility which favors protein aggregation at the interface. ${ }^{32-34}$ On the other hand, interfacial protein layers are weakened if polysaccharides are surface active themselves ${ }^{33}$ or covalently conjugated to adsorbed proteins. ${ }^{35}$ Interfacial rheological measurements with simultaneous subphase exchange presented here facilitate the determination of such complex synergistic and antagonistic biopolymer interactions, as it allows the transient assessment of interfacial moduli and is not limited to measuring final layer properties.

The successive adsorption of a protein and a polysaccharide is exploited in so-called layer-by-layer emulsions. Besides increased interfacial moduli, the secondary polysaccharide layer increases steric repulsion which may enhance emulsion stability. ${ }^{36}$ However, adsorbed polysaccharides may also destabilize emulsions by bridging flocculation or depletion. ${ }^{4,17,34}$ Besides emulsion stability, layer-by-layer adsorption can be employed to create functional emulsions for specific needs. The adsorption of a secondary polysaccharide layer increases heat resistance, ${ }^{2}$ allows the modulation of emulsion zeta-potential, ${ }^{3,4}$ and can prevent the adsorption of enzymes for gastric stable emulsions. ${ }^{6,7}$

\subsection{Multilayer dilatational rheology and structure at the $\mathrm{A} / \mathrm{W}$ interface}

Besides $\mathrm{O} / \mathrm{W}$ interfaces and emulsions, protein-polysaccharide complexes may be employed to improve the stability of foams and respective $\mathrm{A} / \mathrm{W}$ interfaces. ${ }^{37}$ In this section, we characterize $\beta$-lg-pectin multilayers at the $\mathrm{A} / \mathrm{W}$ interface by dilatational rheology and elucidate their structure using neutron reflectivity.

Fig. 3A depicts dilatational amplitude sweeps of pure $\beta$-lg layers compared to multilayers formed by successive and simultaneous pectin injection. All layer showed mostly linear behavior with only a slight decrease in dilatational storage modulus $E^{\prime}$ at increasing deformation. The loss modulus $E^{\prime \prime}$ was lower at all deformation, but was not depicted in Fig. 3A for clarity. The successive electrostatic complexation of pectin resulted in an increase in $E^{\prime}$ compared to the pure $\beta$-lg layer. In contrast, the complexation in the bulk prior to adsorption resulted in lower $E^{\prime}$. Thus, the same effects of simultaneous and successive pectin complexation are observed in dilatational rheology at the $\mathrm{A} / \mathrm{W}$ interface as in shear rheology at the $\mathrm{O} / \mathrm{W}$ 

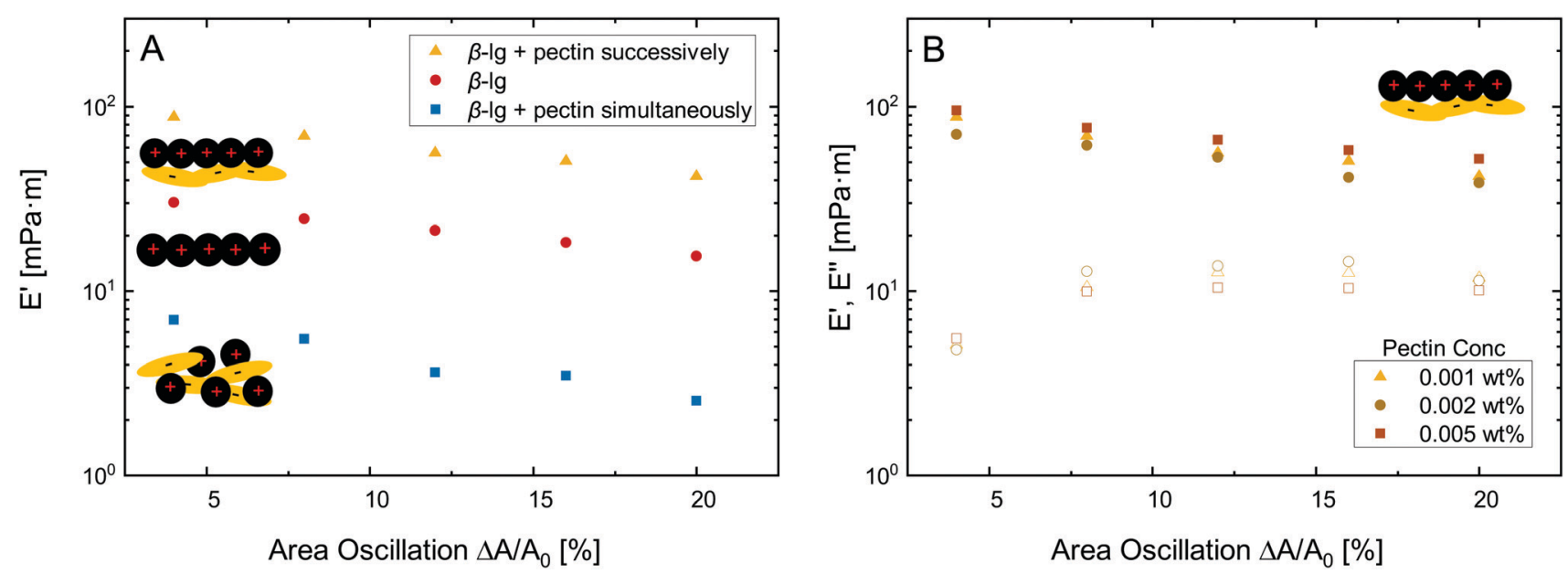

Fig. 3 Dynamic interfacial dilatational storage $E^{\prime}$ (full) and loss modulus $E^{\prime \prime}$ (empty) as a function of area deformation for (A) 0.001 wt\% $\beta$-lg and $0.001 \mathrm{wt} \%$ pectin added simultaneously and successively and (B) increasing pectin concentration added successively. Experiments were performed at the $\mathrm{A} / \mathrm{W}$ interface with $1 / 10 \mathrm{Mcllvaine}$ buffer at $\mathrm{pH} 4$ and $22{ }^{\circ} \mathrm{C}$. Oscillation frequency was $10 \mathrm{mHz}$.

interface discussed above. The interfacial elasticity can not be further enhanced by increasing the concentration of successively added pectin, as demonstrated in Fig. 3B. It is known from interfacial shear rheology that the $\beta$-lg/pectin ratio does not alter the interfacial properties if pectin is added successively. ${ }^{5}$

The adsorption of biopolymer layers at the $\mathrm{A} / \mathrm{W}$ interface further facilitates to probe their structure using neutron reflectivity. Fig. 4 shows the fitted neutron reflectivity, reflectivity relative to the reference $R_{\text {Rel }}$, and scattering length density (SLD) profiles of the respective layers at the air/ $/ \mathrm{D}_{2} \mathrm{O}$ interface.

The biopolymer layers depicted in Fig. 4A were formed in three consecutive steps. (i) A pure $\beta$-lg layer was formed at $0.001 \mathrm{wt} \%$, which was best described by a monolayer with a thickness of $14 \AA$. This corresponds to a loosely packed monolayer, considering the diameter of native $\beta$-lg in solution of approximately $40 \AA$ A. However, protein unfolding and spreading at the interface may result in a more compact $\beta$-lg structure at the interface. ${ }^{10,14}$ Previous studies reported $\beta$-lg monolayers in the range of $20-40 \AA .{ }^{7,38,39}$ The thinner layer formed here can be attributed to a tenfold lower $\beta$-lg concentration, which was chosen to avoid excess $\beta$-lg in the bulk and prevent complexation with pectin. (ii) $0.002 \mathrm{wt} \%$ pectin was successively injected to the adsorbed $\beta$-lg layer. The obtained interfacial layer was best described by a two-layer model consisting of the primary $\beta$ $\lg$ layer followed by the consecutively added pectin. The $\beta$-lg layer was compressed to $11 \AA$ and had a higher surface roughness compared to the initial layer, as denoted by a shift in the SLD profile towards the air phase. Hence, the initial $\beta$-lg layer is compressed and pushed towards the hydrophobic subphase by the consecutive adsorption of pectin. The adsorbed pectin layer was $5 \AA$ in thickness. The thickness of the pectin backbone is $3 \AA{ }^{40}$ indicating a compact pectin layer with limited extension into the water phase. The dense pectin layer is probably the reason why higher pectin concentrations did not increase interfacial dilatational moduli (Fig. 3B). The structure of successive $\beta$-lg-pectin layers may be different in emulsions, as pectins can adsorb at more than one droplet, particularly at high molecular weight. This may induce bridging flocculation, but could also provide additional bulk stabilization. In a third step (iii), the $\mathrm{pD}$ was increased to 7 to investigate if the electrostatic complexation of pectin is reversible upon charge inversion of $\beta$-lg. The obtained layer was indeed best described by a $\beta$-lg monolayer with a thickness of $14 \AA$, same as the initial $\beta$-lg layer. Thus, pectin desorbed due to electrostatic repulsion. The remaining $\beta$-lg layer was rougher compared to the initial layer. It can not be conclusively stated if this derives from irreversible structural changes due to pectin adsorption or traces of remaining pectin. Adsorbed protein layers are known to undergo structural changes at varying $\mathrm{pH}^{15}$ or in presence of like-charged polysaccharides. ${ }^{32-34}$ Thus, interfacial electrostatic $\beta$-lg-pectin complexes are reversible, as previously demonstrated in bulk experiments. ${ }^{27}$ This $\mathrm{pH}$-triggered adsorption and desorption of pectin at protein-stabilized interfaces could allow the production of $\mathrm{pH}$-sensitive colloidal materials. For example, it has been demonstrated that secondary polysaccharide layers can prevent the digestion of proteinstabilized emulsions under gastric conditions. ${ }^{6,7}$ The $\mathrm{pH}$-triggered charge inversion in the duodenum could induce the desorption of this protective layer for controlled delivery applications.

In Fig. 4B the neutron reflectivity of a $\beta$-lg layer formed at $0.001 \mathrm{wt} \%$ is compared to a layer of simultaneously adsorbed $\beta$-lg and pectin at each $0.005 \mathrm{wt} \%$. The $\beta$-lg layer was a $14 \AA$ monolayer as discussed in detail above. Interestingly, the layer formed during simultaneous adsorption of $0.005 \mathrm{wt} \% \beta$-lg and pectin was also best described by a monolayer $14 \AA$ in thickness, and fit goodness increased when the SLD was changed towards the one of $\beta$-lg. This suggests that the interfacial layer formed during simultaneous adsorption consists mostly of $\beta$-lg. The layer roughness was slightly increased similar to the layer after charge inversion at pD 7 discussed above, potentially due to the presence of a minor pectin fraction. Overall, the layer was similar to the one formed with pure $\beta$-lg at fivefold lower concentrations. We conclude that $\beta$-lg-pectin complexes formed 

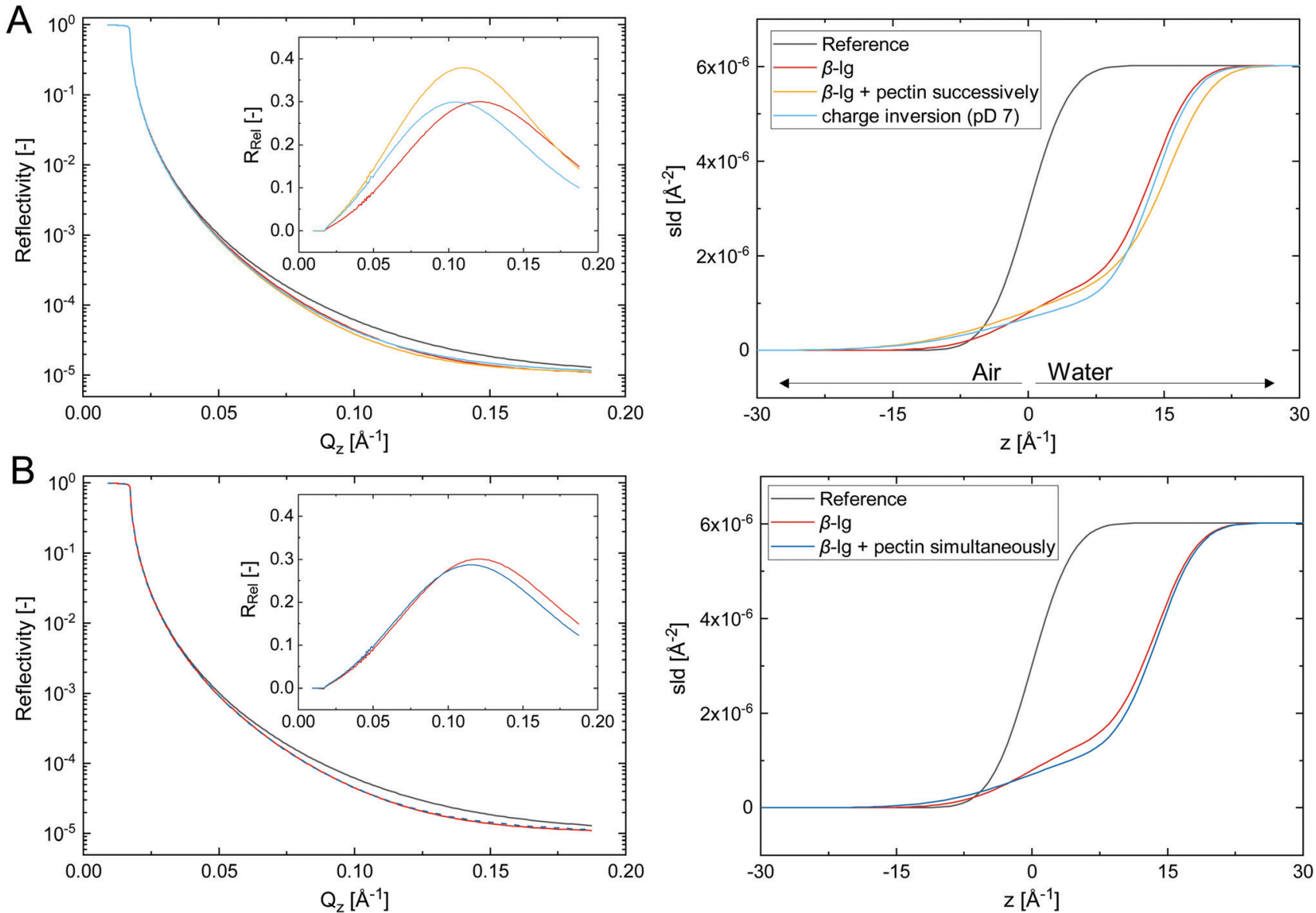

Fig. 4 Fitted neutron reflectivity, reflectivity relative to the reference $R_{\text {Rel, }}$ and scattering length density SLD of biopolymer layers adsorbed at the air/D ${ }_{2} \mathrm{O}$ interface. Experiments were performed in $1 / 10$ Mcllvaine buffer at pD 4 and $20{ }^{\circ} \mathrm{C}$, which was measured as reference. (A) A pure $\beta$-lg layer formed at $0.001 \mathrm{wt} \%$, successive addition of $0.002 \mathrm{wt} \%$ pectin, followed by $\beta$-lg charge inversion by increasing the $\mathrm{pD}$ to 7 . (B) Comparison of a pure protein layer formed at $0.001 \mathrm{wt} \% \beta$-lg and simultaneously adsorbed $\beta$-lg and pectin at $0.005 \mathrm{wt} \%$ each. Reflectivity curves were fitted with the Parratt algorithm and had a $\chi^{2}<7.5 \times 10^{-3}$.

in the bulk show a low tendency towards adsorption, and the complexation reduces the effective $\beta$-lg concentration available for adsorption. Thus, the impeded adsorption kinetics and interfacial viscoelasticity discussed above could derive from a decreased effective protein concentration. This theory is supported by previous findings who demonstrated that the adverse effects of pectin on adsorption and interfacial layer strength can be overcome by increasing the $\beta$-lg concentration. ${ }^{5}$ However, interfacial layers formed at higher $\beta$-lg/pectin ratios also showed a different structure. $^{39}$ A dense $\beta$-lg layer followed by a loosely packed secondary layer of $\beta$-lg-pectin complexes was observed at tenfold higher $\beta-\lg$ concentrations and $\beta-\mathrm{lg} /$ pectin ratios of two to six. This is in contrast to the $\beta-\lg$ dominated monolayer observed here for a mixing ratio of one.

\section{Conclusions}

The formation of protein-polysaccharide multilayers at fluid interfaces is an interesting approach to increase the stability and widen the field of applications of protein-stabilized interfaces. However, the transient measurement of protein-polysaccharide multilayer formation is often difficult. We introduced an interfacial shear rheology setup with simultaneous subphase exchange, allowing the transient measurement of biopolymer multilayers by their viscoelasticity. Thereby, we could demonstrate that the successive adsorption of pectin to an established $\beta$-lg layer increases its interfacial layer strength, whereas simultaneous adsorption impedes adsorption kinetics and viscoelasticity compared to pure $\beta$-lg layers.

Structure analysis by neutron reflectometry confirmed the formation of an initial $\beta$-lg layer followed by electrostatic complexation of a secondary pectin layer. The complexation of pectin was reversible upon $\mathrm{pH}$-induced charge inversion, posing interesting applications for $\mathrm{pH}$-sensitive colloidal materials. For the simultaneous adsorption of $\beta$-lg and pectin we observed a thin monolayer which consists mostly of $\beta$-lg. We concluded that electrostatic complexes decrease the effective protein concentration available for adsorption and show limited surface activity themselves.

\section{Conflicts of interest}

There are no conflicts to declare. 


\section{Acknowledgements}

Neutron reflectivity experiments were performed at the Swiss Spallation Neutron Source SINQ, Paul Scherrer Institute, Villigen PSI, Switzerland. This project was funded by the Swiss National Science Foundation, Project No. 200021-137941 and 200021-175994.

\section{References}

1 V. Mitropoulos, A. Mütze and P. Fischer, Adv. Colloid Interface Sci., 2014, 206, 195-206.

2 T. Harnsilawat, R. Pongsawatmanit and D. J. McClements, J. Agric. Food Chem., 2006, 54, 5540-5547.

3 D. Guzey and D. J. McClements, J. Agric. Food Chem., 2007, 55, 475-485.

4 Y. H. Cho and D. J. McClements, Langmuir, 2009, 25, 6649-6657.

5 R. A. Ganzevles, K. Zinoviadou, T. van Vliet, M. A. Stuart and H. H. De Jongh, Langmuir, 2006, 22, 10089-10096.

6 N. Scheuble, T. Geue, E. J. Windhab and P. Fischer, Biomacromolecules, 2014, 15, 3139-3145.

7 N. Scheuble, M. Lussi, T. Geue, F. Carrière and P. Fischer, Biomacromolecules, 2016, 17, 3328-3337.

8 J. Toro-Sierra, A. Tolkach and U. Kulozik, Food Bioprocess Technol., 2013, 6, 1032-1043.

9 F. MacRitchie and A. E. Alexander, J. Colloid Interface Sci., 1963, 18, 453-457.

10 L. Day, J. Zhai, M. Xu, N. C. Jones, S. V. Hoffmann and T. J. Wooster, Food Hydrocolloids, 2014, 34, 78-87.

11 E. M. Freer, K. S. Yim, G. G. Fuller and C. J. Radke, J. Phys. Chem. B, 2004, 108, 3835-3844.

12 S. Brownlow, J. H. Morais Cabral, R. Cooper, D. R. Flower, S. J. Yewdall, I. Polikarpov, A. C. North and L. Sawyer, Structure, 1997, 5, 481-495.

13 D. Zare, J. R. Allison and K. M. McGrath, Biomacromolecules, 2016, 17, 1572-1581.

14 J. Bergfreund, P. Bertsch, S. Kuster and P. Fischer, Langmuir, 2018, 34, 4929-4936.

15 K. Engelhardt, M. Lexis, G. Gochev, C. Konnerth, R. Miller, N. Willenbacher, W. Peukert and B. Braunschweig, Langmuir, 2013, 29, 11646-11655.

16 E. Dickinson, Soft Matter, 2008, 4, 932-942.

17 E. Dickinson, Food Hydrocolloids, 2011, 25, 1966-1983.
18 P. Fischer, Eur. Phys. J.-Spec. Top., 2013, 222, 73-81.

19 R. A. Ganzevles, M. A. Cohen Stuart, T. van Vliet and H. H. de Jongh, Food Hydrocolloids, 2006, 20, 872-878.

20 P. Erni, P. Fischer, E. J. Windhab, V. Kusnezov, H. Stettin and J. Läuger, Rev. Sci. Instrum., 2003, 74, 4916-4924.

21 P. A. Rühs, N. Scheuble, E. J. Windhab, R. Mezzenga and P. Fischer, Langmuir, 2012, 28, 12536-12543.

22 D. Clemens, P. Gross, P. Keller, N. Schlumpf and M. Könnecke, Phys. B, 2000, 276-278, 140-141.

23 M. Gupta, T. Gutberlet, J. Stahn, P. Keller and D. Clemens, Pramana, 2004, 63, 57-63.

24 L. G. Parratt, Phys. Rev., 1954, 95, 359-369.

25 R. Kohn and P. Kovác, Chem. Zvesti, 1978, 32, 478-485.

26 M. Girard, S. L. Turgeon and S. F. Gauthier, J. Agric. Food Chem., 2003, 51, 6043-6049.

27 B. L. Sperber, M. A. Cohen Stuart, H. A. Schols, A. G. Voragen and W. Norde, Biomacromolecules, 2009, 10, 3246-3252.

28 R. A. Ganzevles, H. Kosters, T. van Vliet, M. A. Stuart and H. H. De Jongh, J. Phys. Chem. B, 2007, 111, 12969-12976.

29 L. Piazza, N. Dürr-Auster, J. Gigli, E. J. Windhab and P. Fischer, Food Hydrocolloids, 2009, 23, 2125-2131.

30 J. N. Miquelim, S. C. Lannes and R. Mezzenga, Food Hydrocolloids, 2010, 24, 398-405.

31 N. P. K. Humblet-Hua, E. van Der Linden and L. M. Sagis, Soft Matter, 2013, 9, 2154-2165.

32 R. Baeza, C. Carrera Sanchez, A. M. Pilosof and J. M. Rodríguez Patino, Food Hydrocolloids, 2004, 18, 959-966.

33 R. Baeza, A. M. R. Pilosof, C. C. Sanchez and J. M. Rodríguez Patino, AIChE J., 2006, 52, 2627-2638.

34 L. S. Jourdain, C. Schmitt, M. E. Leser, B. S. Murray and E. Dickinson, Langmuir, 2009, 25, 10026-10037.

35 T. J. Wooster and M. A. Augustin, Food Hydrocolloids, 2007, 21, 1072-1080.

36 A. Benichou, A. Aserin and N. Garti, J. Dispersion Sci. Technol., 2002, 23, 93-123.

37 E. Dickinson and E. Izgi, Colloids Surf., A, 1996, 113, 191-201.

38 P. J. Atkinson, E. Dickinson, D. S. Horne and R. M. Richardson, J. Chem. Soc., Faraday Trans., 1995, 91, $2847-2854$.

39 R. A. Ganzevles, R. Fokkink, T. van Vliet, M. A. Cohen Stuart and H. H. de Jongh, J. Colloid Interface Sci., 2008, 317, 137-147.

40 T. Imaizumi, M. Szymańska-Chargot, P. M. Pieczywek, M. Chylińska, A. Kozioł, D. Ganczarenko, F. Tanaka, T. Uchino and A. Zdunek, LWT-Food Sci. Technol., 2017, 84, 658-667. 